Original Research Article

\title{
Sinonasal and nasopharyngeal pathology in chronic otitis media patients: a prospective study
}

\author{
Rathwala K.K. ${ }^{1}$ \\ ${ }^{1}$ Dr. Kayedjohar K. Rathwala, Associate Professor, Department of Otorhinolaryngology, Parul Institute of Medical Sciences and \\ Research Centre, Vadodara, Gujarat, India
}

Corresponding Author: Dr. Kayedjohar K. Rathwala, Department of Otorhinolaryngology, Parul Institute of Medical Sciences and Research Centre, Vadodara, Gujarat, India. Email Id: drkayedjohar@yahoo.co.in

\begin{abstract}
Background and Aim: Chronic Suppurative Otitis Media (CSOM) is a persistent inflammation that causes irreversible changes of the mucosa in the middle ear and mastoid cavity. The present study was conducted at ENT department of Tertiary care institute of Gujarat, India involving 130 patients suffering from chronic otitis media. The study also aimed to compare the microbiological flora of the diseased ear and the nasopharynx. Material and Methods: Present prospective study conducted at department of Otorhinolaryngology Tertiary care institute of Gujarat. Total 130 patients having a clinical diagnosis of chronic suppurative otitis media were invited to participate in the study and an informed consent was sought. Clinical presentation including complaints related with ear and nose were noted. Bilateral ear examination was done using Valsalva maneuver. Pure tone audiometry was also performed on all the cases to assess the effect on hearing status. The nasopharyngeal swab was collected by a sterile technique to avoid commensal growth. Results: The most common presenting nasal complaint was nasal obstruction, followed by postnasal drip, nasal discharge, sneezing, snoring, headache, around $25 \%$ of patients had no nasal symptoms. The comparative study of ear and nasopharyngeal swab showed statistically significant correlation. $80 \%$ of ear swabs and $92 \%$ of nasopharyngeal swabs were found to be sterile. Conclusion: Chronic otitis media is a disease with multiple factors and different possible aetiologies. The concept of a relationship between sinonasal and nasopharyngeal pathologies and chronic otitis media has been supported by the present study.
\end{abstract}

Keywords: Chronic Suppurative Otitis Media, Nasal obstruction, Nasopharyngeal swab, Sinonasal

\section{Introduction}

Chronic Suppurative Otitis Media (CSOM) is a persistent inflammation that causes irreversible changes of the mucosa in the middle ear and mastoid cavity. It is characterized by a persistent discharge from the middle ear through a tympanic membrane perforation. It is an important cause of preventable hearing loss, particularly in the developing world [1]. Its pathophysiology begins with irritation and subsequent inflammation of the middle ear mucosa.

The most important pathological factors in CSOM are dysfunction of the Eustachian tube and bacterial infection. Recurrent attacks of acute otitis media and eustachian tube dysfunction are few among those. Pathologies involving nasopharynx and paranasal sinuses can lead to dysfunction of eustachian tube due to its close anatomical relationship. A relationship between allergic rhinitis and otitis media has been postulated since many years. Improvement of otitis

Manuscript received: $25^{\text {th }}$ October 2019

Reviewed: $4^{\text {th }}$ November 2019

Author Corrected: $10^{\text {th }}$ November 2019

Accepted for Publication: $16^{\text {th }}$ November 2019

Tropical Journal of Ophthalmology and Otolaryngology media following septoplasty was also noted and evidenced by few authors in their study. Bozkus et al $[2,3]$ demonstrated that the role of sinonasal abnormalities and allergic rhinitis in the pathogenesis of chronic otitis media is prevalent.

They concluded that although medical history and physical examination are considered mandatory procedures during the initial evaluation of the patients with chronic otitis media, radiological, endoscopic, and other auxillary diagnostic tools should be used in the objective assessment of the patients, and lesions of the nasal cavity, and the nasopharynx must be always taken into consideration in the differential diagnosis.

The present study was conducted at ENT department of Tertiary care institute of Gujarat, India involving 130 patients suffering from chronic otitis media.

The study also aimed to compare the microbiological flora of the diseased ear and the nasopharynx. 


\section{Material and Methods}

Type of study: Present prospective study was conducted at the department of Otorhinolaryngology Tertiary care institute of Gujarat. The study period comprised of 1.5 years.

Sampling Methods: Total 130 patients having a clinical diagnosis of chronic suppurative otitis media were invited to participate in the study and an informed consent was sought. All the patients providing consent to participate in the study were enrolled as study subjects.

After enrolment in the study, demographic data of the patient was noted on a semi-structured proforma prepared for the purpose. Clinical presentation including complaints related with ear and nose were noted.

Inclusion criteria: All patients willing for the study with chronic ear discharge- both unilateral \& bilateral and only those patients who gave consent to participate in the study were included.

Exclusion criteria: Exclusion criteria were traumatic perforation; acute infection of nose and PNS; congenital condition- cleft palate, choanal atresia; post sinonasal and nasopharyngeal surgeries; co morbid conditions- diabetes mellitus.
The patients were then subjected to diagnostic nasal endoscopic examination of both the sides. Bilateral ear examination was done using Valsalva maneuver. Pure tone audiometry was also performed on all the cases to assess the effect on hearing status.

The nasopharyngeal swab was collected by a sterile technique to avoid commensal growth. A specially designed long tipped sterile swab was used. It was introduced into a sterile suction catheter. This avoided the contact between the swab and the nasal mucosa.

The catheter with the swab was introduced into the nasal cavity under endoscopic guidance and soon after reaching the choana, the swab alone was pushed further inside to contact the nasopharyngeal mucosa at the region of eustachian tube orifice.

After 10 seconds swab was withdrawn into the catheter and was removed out of the nasal cavity. It was then sent for culture and sensitivity in a sterile container.

Statistical analysis: The data were analyzed using SPSS version 15 (SPSS Inc., Chicago, Illinois, USA). For all tests, confidence level and level of significance were set at $95 \%$ and $5 \%$ respectively.

\section{Results}

A total of hundred and thirty patients with chronic otitis media were studied. Out of which 105 patients were suffering from mucosal and 25 patients from squamosal type of otitis media. The most common presenting nasal complaint was nasal obstruction, followed by postnasal drip, nasal discharge, sneezing, snoring, headache, around $25 \%$ of patients had no nasal symptoms.

The distribution of nasal symptoms is shown in Table 1. The most common otoscopic findings in a mucosal type of COM were a subtotal central perforation which was $28 \%$ in $\mathrm{U} / \mathrm{L}$ and $6 \%$ in $\mathrm{B} / \mathrm{L}$ cases.

The most common otoscopic finding in a squamosal type of COM was a postero superior retraction pocket with granulations seen in $13 \%$ of cases.

Table-1: Nasal symptoms among study participants.

\begin{tabular}{|c|c|c|}
\hline Symptom & Number of Cases & Percentages \\
\hline Nasal obstruction & 71 & 54.6 \\
\hline Nasal discharge & 27 & 20.7 \\
\hline Sneezing & 20 & 15.3 \\
\hline Postnasal drip & 25 & 19.2 \\
\hline Headache & 10 & 7.6 \\
\hline Snoring & 13 & 10 \\
\hline No symptoms & 33 & 25.3 \\
\hline
\end{tabular}


Original Research Article

Table-2: Microbiological study of ear and nasopharyngeal swab.

\begin{tabular}{|c|c|c|c|c|c|c|c|c|}
\hline & \multicolumn{7}{|c|}{ Bacteriological study of nasopharyngeal swab } \\
\hline & & $\begin{array}{l}\mathrm{Co}- \\
\mathrm{SA}\end{array}$ & $\begin{array}{c}\text { KL. } \\
\text { Pneumoniae }\end{array}$ & MRSA & MSSA & Sterile & $\begin{array}{c}\text { Streptococci } \\
-\mathbf{a}\end{array}$ & Total \\
\hline \multirow{7}{*}{$\begin{array}{l}\text { Bacteriological } \\
\text { study of ear swab }\end{array}$} & ASP sp & 0 & 0 & 0 & 0 & 1 & 0 & 1 \\
\hline & Kl. pneumoniae & 0 & 0 & 0 & 0 & 2 & 0 & 2 \\
\hline & MSSA & 1 & 0 & 0 & 4 & 4 & 0 & 9 \\
\hline & Pseudomonas & 0 & 1 & 1 & 0 & 10 & 0 & 12 \\
\hline & Sterile & 0 & 0 & 1 & 0 & 103 & 0 & 104 \\
\hline & Enterococci & 0 & 0 & 0 & 0 & 0 & 2 & 2 \\
\hline & Total & 1 & 1 & 2 & 4 & 123 & 2 & 130 \\
\hline
\end{tabular}

The comparative study of ear and nasopharyngeal swab showed statistically significant correlation. $80 \%$ of ear swabs and $92 \%$ of nasopharyngeal swabs were found to be sterile. The results were compared by chi square tests and were found to be statistically significant $(\mathrm{p} \leq 0.05)$. Around $7 \%$ of ear cases showed MSSA growth and $3 \%$ cases from nasopharynx of corresponding side also grew the same organism. Out of which $3.5 \%$ were in concurrence with the study which was also statistically significant. Pseudomonas aueroginosa was grown in $9.2 \%$ of ear swabs whereas their corresponding nasopharyngeal swabs were sterile (Table 2). Those cases were squamosal type of COM.

\section{Discussion}

The first step in diagnosis of chronic suppurative otitis media is to understand its underlying pathology, once underlying pathology is identified; it is easy to offer treatment for the disease. Identification and understanding of nasopharyngeal and sinonasal pathology of the disease helps to locate and understand the magnitude of the disease that helps in successful management of the disease. Higher incidence of COM is common in cases due to the malnutrition, overcrowding and poor hygiene and hence is more commonly seen to occur in communities from poor socio-economic standards [2].

Age of patients ranged from 9 to 69 years with a mean age of $28.14 \pm 09.25$ years. Maximum number of patients (40\%) was aged between 11-20 years. CSOM is defined primarily as a disease of pediatric age group [4]. Other workers like Shrestha et al [5], Karki et al [6], Poorey and Iyer [7] also found maximum prevalence of CSOM in the younger age group.

In the present study patients presented with various combinations of nasal and sinus symptoms. The most common presenting nasal complaint was nasal obstruction followed by postnasal drip, nasal discharge and other sinonasal symptoms. Most of the patients had combination of two or more symptoms. These nasal symptoms were also chronic in nature and not all were present at the time of data collection. In a study conducted by Singh et al unilateral nasal obstruction with or without discharge was one of the most common symptom (59\%), which was consistent with the present study [8]. Among different sinonasal pathologies deviated nasal septum (DNS) was the most common finding. It was found to be present in a total of 61 cases, it was a solitary finding in 28 cases. These findings are in agreement with the findings of Yeolekar et al and Bozkus et al who also observed that deviated septum alone or in combination was the most common sinonasal pathology [7,9]. These findings are in agreement with the findings of Yeolekar et al [10] and Bozkus et al [3] who also observed that deviated septum alone or in combination was the most common sinonasal pathology.

After deviated nasal septum, sinusitis was the next common finding, which was diagnosed based on the symptoms such as postnasal drip, headache with or without nasal obstruction and signs on nasal endoscopy such as Concha bullosa, prominent agger nasi. Fujita et al reported sinusitis to be present in $48 \%$ cases of refractory otitis media [11]. Gopalakrishnan and Kumar reported sinusitis to be a major pathology of COM in adult subjects [12].

After DNS, allergic rhinitis was the most common finding. Bozkuset $\mathrm{F}$ al [3] demonstrated that the role of sinonasal abnormalities and allergic rhinitis in the pathogenesis of chronic otitis media is prevalent. Ghonaim et al [13] also highlighted allergic rhinitis to be one of the important predictors of CSOM. Rhinitis is influential in the occurrence of CSOM via two mechanisms which include Eustachian tube dysfunction secondary to allergic reactions effective on nasal mucosa and decrease in the frequency of 


\section{Original Research Article}

ciliary whipping motions [14]. In the present study, the microbiological flora of both ear and corresponding side of nasopharynx were compared. Since acute infections were excluded from the study, majority of swabs from the ear and nasopharynx were found to be sterile. $80 \%$ of ear swabs and $92 \%$ of nasopharyngeal swabs showed no growth.

Fireman et al [15] emphasized that otitis media is a multifactorial disease which was effected by many etiologies including nasal, and paranasal sinus abnormalities.

The present study confirmed these observations. Bluestone et al [16] were of the view that that diseases of the sinuses are the main cause of CSOM rather than adenoid in adolescents and adults for Eustachian tube block.

Otitis media is a multifactorial disease which is affected by many aetiologies including nasal, nasopharyngeal and paranasal sinus abnormalities and the present study confirmed these observations.

As this was prospective study with limited sample size results cannot be generalized to the whole country population. This study can thus be followed up by a cohort study to know the precise Sino nasal pathologies.

\section{Conclusion}

Chronic otitis media is a disease with multiple factors and different possible aetiologies. The concept of a relationship between sinonasal and nasopharyngeal pathologies and chronic otitis media has been supported by the present study. Sino nasal pathologies are the major risk factor for CSOM and in order to treat the CSOM, first the sino nasal pathologies should be ruled out and should be addressed first followed by ear pathology.

\section{What the study adds to the existing knowledge?}

Proper diagnostic nasal evaluation is necessary in all cases of COM for comprehensive management of the disease. Sinonasal diseases being one of the important foci of infection leading to chronic otitis media, its detection and management is equally important as the ear surgery per se.

Hence all patients with COM should undergo a thorough sinonasal and diagnostic nasal endoscopic examination and their pathology should be ruled out or cleared before any surgical management of ear disease to be undertaken.

\section{Author's contribution}

Dr. Kayedjohar K. Rathwala formulated the aims \& objectives with study design, data collection from medical record department, preparation of the manuscript and data analysis.

Funding: No funding sources

Conflict of interest: None declared

Ethical Approval:This study was approved by the Institutional Ethics Committee

\section{References}

1. WHO. Chronic suppurative otitis media: burden of illness and management options, World Health Organization, Geneva, Switzerland, 2004.

2. Grady D, Mathias P, Anderson R, Snider G, Sprinkle PM. Improvement of middle ear disease following septoplasty. Otology Neurotol. 1983;4(4):327-331.

3. Bozkus F, Bozan NA, Iynen IS, Sakin YF, Kiris M. Analysis of sinonasal, pharyngeal and allergy-related risk factors for chronic suppurative otitis media. Acta Medica Mediterranea. 2013;29:47-52.

4. Nelson JD. Chronic suppurative otitis media. Pediatr Infect Dis J. 1988;7(6):446-448.

5. Shrestha D, Thapa P, Bhandari YB. Types of pathology and ossicular status in atticoantral disease undergoing mastoidectomy at Bir Hospital. J Col Med Sci-Nepal. 2010;6(4):26-30. doi: https://doi.org/10.3126/jcmsn. v6i4. 6722 .

6. Karki R, Rai K. Pattern of Otorhinolaryngological Diseases at Rural Medical Camps in Far Western Region of Nepal. Med J Shree Birendra Hos. 2012;11(2):29-31. doi: https://doi.org/10.3126/mjsbh.v11i2.7906.

7. Poorey VK, Lyer A. Study of bacterial flora in csom and its clinical significance. Indian J Otolaryngol Head Neck Surg. 2002; 54(2): 91-95. doi: 10. 1007/ BF02968724.

8. Adoga A, Nimkur T, Silas O. Chronic suppurative otitis media: Socio-economic implications in a tertiary hospital in Northern Nigeria. Pan Afr Med J. 2010;4:3. doi: 10. 4314/ pamj. v4i1.53613.

9. Sharma K, Aggarwal A, Khurana PM. Comparison of bacteriology in bilaterally discharging ears in chronic suppurative otitis media. Indian J Otolaryngol Head Neck Surg. 2010; 62(2):153-157. doi: 10.1007/s12070-010-00219. Epub 2010 Sep 24.

10. Yeolekar AM, Dasgupta KS. Otitis media: Does the onus lie on sinonasal pathology. Indian J Otol. 2011; 17(1): 8-11. doi: 10.4103/0971-7749.85784. 


\section{Original Research Article}

11. Dayasena R, Dayasiri M, Jayasuriya C, Perera D. Aetiological agents in chronic suppurative otitis media in Sri Lanka. Australas Med J. 2011;4(2):101-104. doi: 10. 4066/ AMJ.2011.549. Epub 2011 Feb 28.

12. Shyamala R, Reddy PS. The study of bacteriological agents of chronic suppurative otitis media-aerobic culture and evaluation. J Microbiol Biotech Res. 2012; 2(1): 152-162.

13. Ghonaim MM, El-Edel RH, Bassiony LM, Alzahrani SS. Otitis Media in Children: Risk Factors \& Causative Organisms. Ibnosina J Med Biomed Sci. 2011;3(5): 172-181.
14. Hurst DS. The role of allergy in otitis media with effusion. Otolaryngol Clin North Am. 2011;44(3):637-654, viii-ix. doi: 10.1016/j.otc.2011.03.009. Epub 2011 May 2.

15. Fireman P. Otitis media and nasal disease: a role for allergy. J Allergy Clin Immunol. 1988;82(5 Pt 2):917-926. doi: 10.1016/0091-6749(88)90034-6.

16. Blue Stone CD. Assessment of Eustachian tube function. In Jerger $J(E d)$ : Handbook of clinical impedance Audiometry, New York, American Electormedics Corporation, 1975, 127-148.

\section{How to cite this article?}

Rathwala K.K. Sinonasal and nasopharyngeal pathology in chronic otitis media patients: a prospective study. Trop J Ophthalmol Otolaryngol.2019;4(7):409-413. doi:10.17511/jooo.2019.i07.02 